\title{
Asymmetric Two-Terminal Graphene Detector for Broadband Radiofrequency Heterodyne- and Self-Mixing
}

Jiayue Tong, ${ }^{\dagger}$ Matthew C. Conte,,+ Thomas Goldstein, ${ }^{\dagger}$ Sigfrid K. Yngvesson, ${ }^{*}$ Joseph C. Bardin ${ }^{+}$and Jun Yan ${ }^{+*}$

${ }^{\dagger}$ Department of Physics, University of Massachusetts, Amherst, Massachusetts 01003, USA

Department of Electrical and Computer Engineering, University of Massachusetts, Amherst, Massachusetts 01003, USA

"Corresponding Author: Jun Yan. Tel: (413)545-0853 Fax: (413)545-1691

E-mail: yan@physics.umass.edu 


\begin{abstract}
Graphene, a single atomic layer of covalently bonded carbon atoms, has been investigated intensively for optoelectronics and represents a promising candidate for high-speed electronics. Here we present a microwave mixer constructed as an asymmetricallycontacted two-terminal graphene device based on the thermoelectric effect. We report a 50 $\mathrm{GHz}$ (minimum) mixer bandwidth as well as $130 \mathrm{~V} / \mathrm{W}(163 \mathrm{~mA} / \mathrm{W})$ extrinsic directdetection responsivity. Anomalous second-harmonic generation due to self-mixing in our graphene detector is also observed. Careful investigation of the responsivity from four different approaches gives consistent results, confirming the exceptional performance of our zero-bias device operating at room temperature. The $50 \mathrm{GHz}$ bandwidth indicates an extremely fast response time and our experimental results represent an encouraging advance towards practical graphene microwave devices, with anticipated future applications extended through millimeter wave and terahertz frequencies.
\end{abstract}

Key words: graphene detector, microwave mixer, RF heterodyne receiver, secondharmonic generation, thermoelectric effect, bandwidth 


\section{Manuscript text}

The interest in graphene has been rapidly growing in recent years with numerous demonstrations showing its usefulness as a practical industrial material. The roll-to-roll mass production of this layer-by-layer transferrable two-dimensional atomically thin sheet, ${ }^{1}$ together with its unique properties, ${ }^{2}$ make graphene highly attractive for integration with various waveguide technologies, silicon compatible integrated circuits, as well as large area flexible electronics. ${ }^{3-7}$ A highly promising area of application for graphene is high-frequency large-bandwidth electronics based on characteristics such as its high intrinsic carrier mobility, high carrier saturation velocity, ultra-short response time and ambipolar charge transport. ${ }^{8-10}$

Graphene radiofrequency (RF) mixers and harmonic multipliers have been explored for about a decade. ${ }^{5,10-16}$ Initial graphene mixers demonstrated the feasibility of designing such devices, ${ }^{10}$ but had performance data that were orders-of-magnitude away from values required in applications. ${ }^{9}$ The graphene field effect transistor ("GFET") configuration dominated the gradual development of graphene mixers through the $\mathrm{GHz}$ to hundreds of $\mathrm{GHz}$ frequency range. ${ }^{5,10-16}$ At low frequencies, GFET mixers and multipliers still cannot compete with the dominant CMOS and other semiconductor technologies. However, at millimeter wave frequencies recent GFET mixers with conversion loss of 29 $\mathrm{dB}$ and bandwidth of $15 \mathrm{GHz}^{14}$ have begun to demonstrate comparable performances. This type of IC could be useful in the future development of high speed communication systems around $200 \mathrm{GHz} .{ }^{17}$ At even higher frequencies, the GFET mixers have higher conversion loss of $60 \mathrm{~dB}$ at an LO power of $-10 \mathrm{dBm}$, and smaller bandwidth of $5 \mathrm{GHz},{ }^{16}$ suggesting 
that the FET technology has problems due to parasitic reactance at these higher frequencies. Note that the GFET microwave and millimeter-wave mixers studied so far have consistently smaller bandwidth than what the intrinsic graphene speed has to offer. ${ }^{5,12,14,16}$ In contrast, applications in high-speed (i.e. wide-bandwidth) data communications utilizing fiber and waveguide based optical systems, which take advantage of the ability of graphene to absorb and detect a very wide range of infrared photons at speeds higher than $60 \mathrm{GHz}$, have advanced more rapidly. ${ }^{6,7,18-20}$

In this Letter, we use a two-terminal asymmetrically-contacted graphene device to realize a high-performance RF heterodyne mixer with low conversion loss and very broad bandwidth, exceeding $50 \mathrm{GHz}$, which is the upper frequency limit of our present measurement system. Such a wide bandwidth is unprecedented for graphene RF mixers and represents an encouraging development upon several previous mixer designs whose best bandwidth was limited to $15 \mathrm{GHz} .{ }^{5,12,14,16}$ Further, since the thermoelectric (TE) effect harnessed in the device is essentially frequency independent, we can extrapolate the performance of the TE mixer to millimeter wave and $\mathrm{THz}$ frequencies, which, combined with the highly-desirable broad bandwidth, may find useful future applications in emerging areas of high speed data communication and (sub)millimeter wave imaging. ${ }^{17,21}$ We further found that our graphene device is highly efficient in generating second-harmonic signals. This is surprising because as a centrosymmetric material, graphene's second-order nonlinear susceptibility $\chi^{(2)}$ is expected to be zero. ${ }^{22}$ We interpret our second-harmonic signal as being the result of RF self-mixing, due to the asymmetric contacts of our device. This interpretation is confirmed by a control sample with one order of magnitude smaller asymmetry, which correspondingly produces a second-harmonic signal that is 
approximately two orders of magnitude weaker.

a

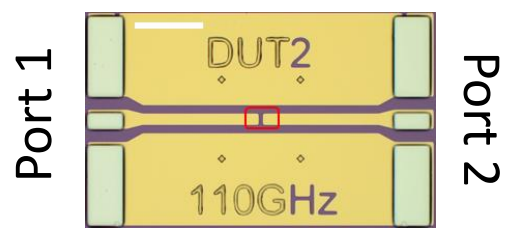

b

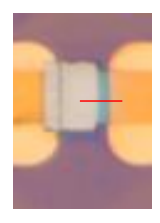

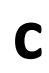

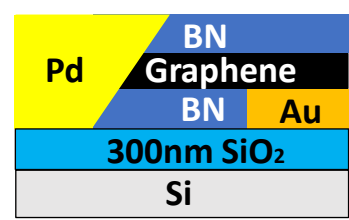

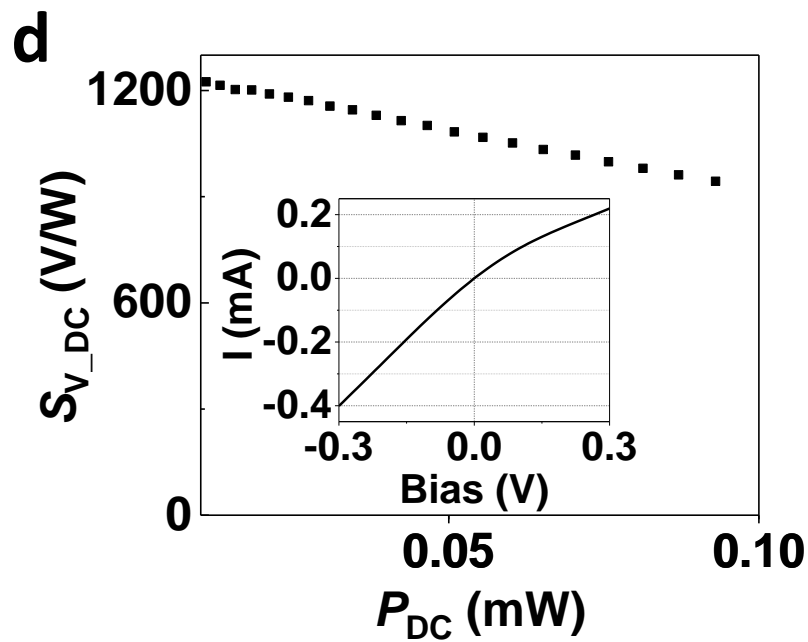

Figure 1. (a) Picture of the coplanar waveguide (CPW). The white scale bar is 100 $\mu \mathrm{m}$. The red rectangle is the area where graphene is transferred to. (b) Zoomedin view of the BN/graphene/BN piece transferred to the CPW. (c) Schematic sideview of the graphene device indicated by the red line in (b). (d) Responsivity calculated from the I-V curve. Inset: I-V curve of the device.

Our RF mixer device is made by dry-transfer of hexagonal boron nitride (hBN) protected graphene to the signal line of a pre-fabricated GSG (ground-signal-ground) coplanar waveguide (CPW; Fig. 1a). To break the mirror symmetry of the device, one of the two terminals is etched with $\mathrm{CHF}_{3}$ and $\mathrm{O}_{2}$ plasma, and subsequently contacted from the edge by palladium (Pd) sputtering (more fabrication details in Methods). ${ }^{23}$ Figure $1 \mathrm{~b}$ shows the optical microscope image of the sample, and the schematic side view is illustrated in Fig. 1c. Comparing with several other different contact strategies we explored before ${ }^{24}$ such as Au edge source / Pd top drain, Au edge source / Au top drain and Au top source / Pd top drain, we found that this combination gives lower impedance while 
maintaining high responsivity.

We first characterize the asymmetry of our device by performing DC charge transport measurements. As shown in the inset of Fig. 1d, the $I-V$ curve of our device is nonlinear and asymmetric under positive and negative biases. This asymmetry arises from the thermoelectric response of the graphene device under DC current heating, which creates a hot spot in the center of the graphene sample and a temperature gradient towards the metal-contact heat sinks. The non-uniform temperature profile along the device and the asymmetric Fermi energy distribution due to the different contacts create a non-zero thermoelectric voltage superimposed on the applied bias voltage. ${ }^{24,25} \mathrm{We}$ make use of the $I-V$ curve to extract the TE voltage responsivity of the device. When the sample is biased with a positive voltage $V$, the current $I_{+}$in the sample is given by $V / R_{\mathrm{G}}+I_{\mathrm{th}}$, where $R_{\mathrm{G}}$ is the resistance of the sample (about $800 \Omega$ for this device) and $I_{\text {th }}$ is the thermoelectric current. When the bias voltage changes sign, so does the bias current, while $I_{\text {th }}$ remains in the same direction which is pre-determined by the sample asymmetry produced during device fabrication, i.e. $I_{-}=-V / R_{\mathrm{G}}+I_{\mathrm{th}}$. The thermoelectric voltage responsivity of the device can then be calculated as

$$
S_{\mathrm{V}_{-} \mathrm{DC}}=I_{\mathrm{th}} R_{\mathrm{G}} / P_{\mathrm{DC}}
$$

where $P_{\mathrm{DC}}=V^{2} / R_{\mathrm{G}}$ is the heating power. Figure $1 \mathrm{~d}$ main panel shows the responsivity of the device as a function of $P_{\mathrm{DC}}$, which is about $1000 \pm 200 \mathrm{~V} / \mathrm{W}$; a similar magnitude of responsivity has been observed before in other graphene TE devices. ${ }^{24,25}$

The TE voltage can be generated by other means of heating. For example, the graphene TE detector is a promising platform for sensing in the challenging THz band. ${ }^{24,25}$ Here we focus on the RF response of the graphene TE detector. We couple microwave 
(MW) radiation capacitively through two bias-tees connected to the graphene device (Fig. 2a inset; more details in Methods). The TE voltage difference $V_{\mathrm{d}}$ between the graphene source and drain leads is read out from the inductive ports of the bias-tees. To reduce standing waves in the circuit, a $10-\mathrm{dB}$ attenuator is inserted after the MW source. We calibrated and measured the input MW power $P_{\mathrm{MW}}$ right after the input bias-tee. The measured $V_{\mathrm{d}}$ then allows us to extract the extrinsic MW responsivity of our device $S_{\mathrm{V}_{-} \mathrm{MW}}$ $=V_{\mathrm{d}} / P_{\mathrm{MW}}$. Figure 2a shows that the device responsivity to $1 \mathrm{GHz} \mathrm{MW}$ heating is about 130 $\pm 10 \mathrm{~V} / \mathrm{W}$ (more data for measurements up to $50 \mathrm{GHz}$ are shown in the Supporting Information). We note that the observed $S_{\mathrm{V}_{-} \mathrm{MW}}$ in Fig. 2a is much smaller than $S_{\mathrm{V}_{-} \text {DC }}$ in Fig. 1d under DC heating. This can be understood as a result of impedance mismatch; the graphene load resistance $R_{\mathrm{G}}=800 \Omega$ is much larger than $50 \Omega$ and a significant portion of the MW power is reflected rather than delivered to the graphene. The mismatch loss can be estimated by $-10 \log _{10}\left[1-\left(\frac{800-50}{800+50}\right)^{2}\right] \approx 6.5 \mathrm{~dB}$. Experimentally we quantify the system loss by performing $S$-parameter measurements using a vector network analyzer (VNA, Keysight N5247A; the measured $S_{11}, S_{12}, S_{22}$ and $S_{21}$ are shown in Supporting Information). The mismatch loss of the system is given by $-10 \log _{10}\left(1-\left|S_{11}\right|^{2}-\left|S_{21}\right|^{2}\right)$ for port 1 , and by $-10 \log _{10}\left(1-\left|S_{22}\right|^{2}-\left|S_{12}\right|^{2}\right)$ for port 2 . Figure $2 b$ shows the measured mismatch loss of our sample, which ranges between 8 and $4 \mathrm{~dB}$ from low frequency to 50 $\mathrm{GHz}$, consistent with the $6.5 \mathrm{~dB}$ estimation from the sample DC resistance. Taking into account the $7.5 \mathrm{~dB}$ mismatch loss at $1 \mathrm{GHz}$, the intrinsic responsivity of our detector is then estimated to be $730 \pm 60 \mathrm{~V} / \mathrm{W}$, which is in reasonable agreement with the results of our DC measurement in Fig. 1d. 


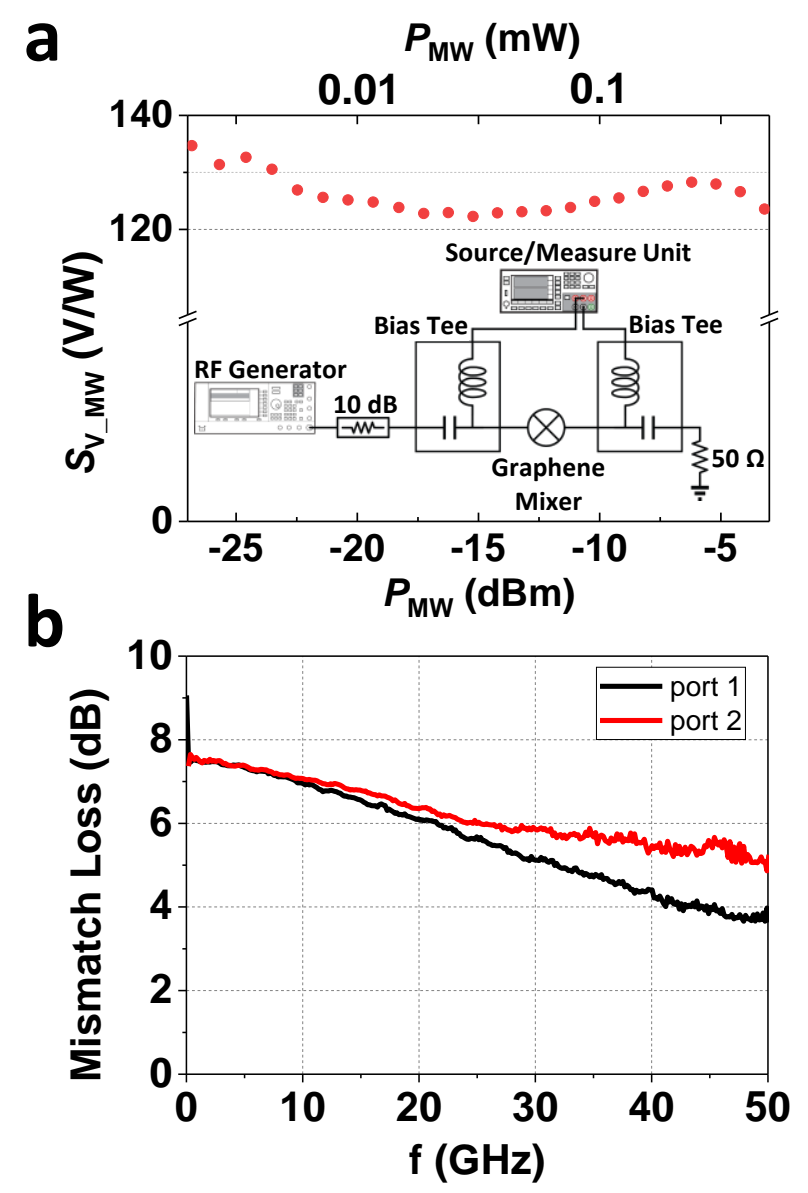

Figure 2. (a) Power dependence of direct-detection extrinsic responsivity at 1 GHz. Inset: Microwave direct detection set-up. (b) Mismatch loss of the device calculated from the $S$ parameters.

We note that the extrinsic responsivity $S_{\mathrm{V} \_ \text {ext }}$ of $\sim 130 \mathrm{~V} / \mathrm{W}$ or $163 \mathrm{~mA} / \mathrm{W}$ in Fig. $2 \mathrm{a}$ is among the highest observed for graphene high-speed direct-power detectors. In the infrared range, earlier versions of graphene photodetectors for optical communication had $S_{\mathrm{V}_{-} \text {ext }}$ of $6.1 \mathrm{~mA} / \mathrm{W},{ }^{18}$ and later improvements by integration with waveguides reached 35 to $100 \mathrm{~mA} / \mathrm{W} \cdot \cdot^{6,720}$ In the $\mathrm{GHz}-\mathrm{THz}$ frequency range, we previously demonstrated a graphene TE detector with a $S_{\mathrm{V}_{-} \text {ext }}$ of $4.9 \mathrm{~V} / \mathrm{W}$ at $1.9 \mathrm{THz} .{ }^{24}$ An earlier work using a gated GFET reached $14 \mathrm{~V} / \mathrm{W}$ at $600 \mathrm{GHz} .{ }^{26}$ A more recent GFET design obtained a responsivity 
of $74 \mathrm{~V} / \mathrm{W}$ at a frequency of $400 \mathrm{GHz} \cdot{ }^{15} \mathrm{We}$ anticipate that with future improvements in impedance mismatch, the simple two-terminal graphene device presented here may even become competitive with high-performance diode devices. ${ }^{27}$

The RF detection in Fig. 2 as well as the (sub)THz detections in Refs.15,24,26 yield a DC voltage output (direct power detection) which erases the frequency information of the input signal. We infer from the high response speed of graphene $e^{18,25,28,29}$ that it should be possible to make a heterodyne RF detector with very large bandwidth, where the RF signal is mixed with a local oscillator (LO), creating radiation at an intermediate frequency (IF) as either an upper side band (USB) or a lower side band (LSB) signal. In this mode of detection, the frequency information of the incident RF signal is retained: $f_{I F}^{U S B}=f_{L O}+$ $f_{R F}$ and $f_{I F}^{L S B}=\left|f_{L O}-f_{R F}\right|$. RF heterodyne mixers have been demonstrated before with graphene; these are typically three terminal FET devices in which the RF and LO are applied to the gate and the drain separately. ${ }^{5,12,14}$ Our design is different in that it is a twoterminal asymmetric device and both RF and LO are applied to the drain electrode. One advantage of this design is the reduction of parasitic capacitance which consequently makes the TE device suitable for achieving much larger bandwidth.

Figure 3a shows our experimental setup for the heterodyne detection. We use a 3 dB RF directional coupler to feed the outputs of two Keysight E8257D analog signal generators into the graphene device through a $10-\mathrm{dB}$ attenuator; one at $f_{L O}$ serving as the local oscillator and the other at $f_{R F}$ as the RF signal. The IF outputs from the graphene device, as well as the transmitted LO and RF, are measured with a calibrated Keysight PXA spectrum analyzer (see Methods for more details). 

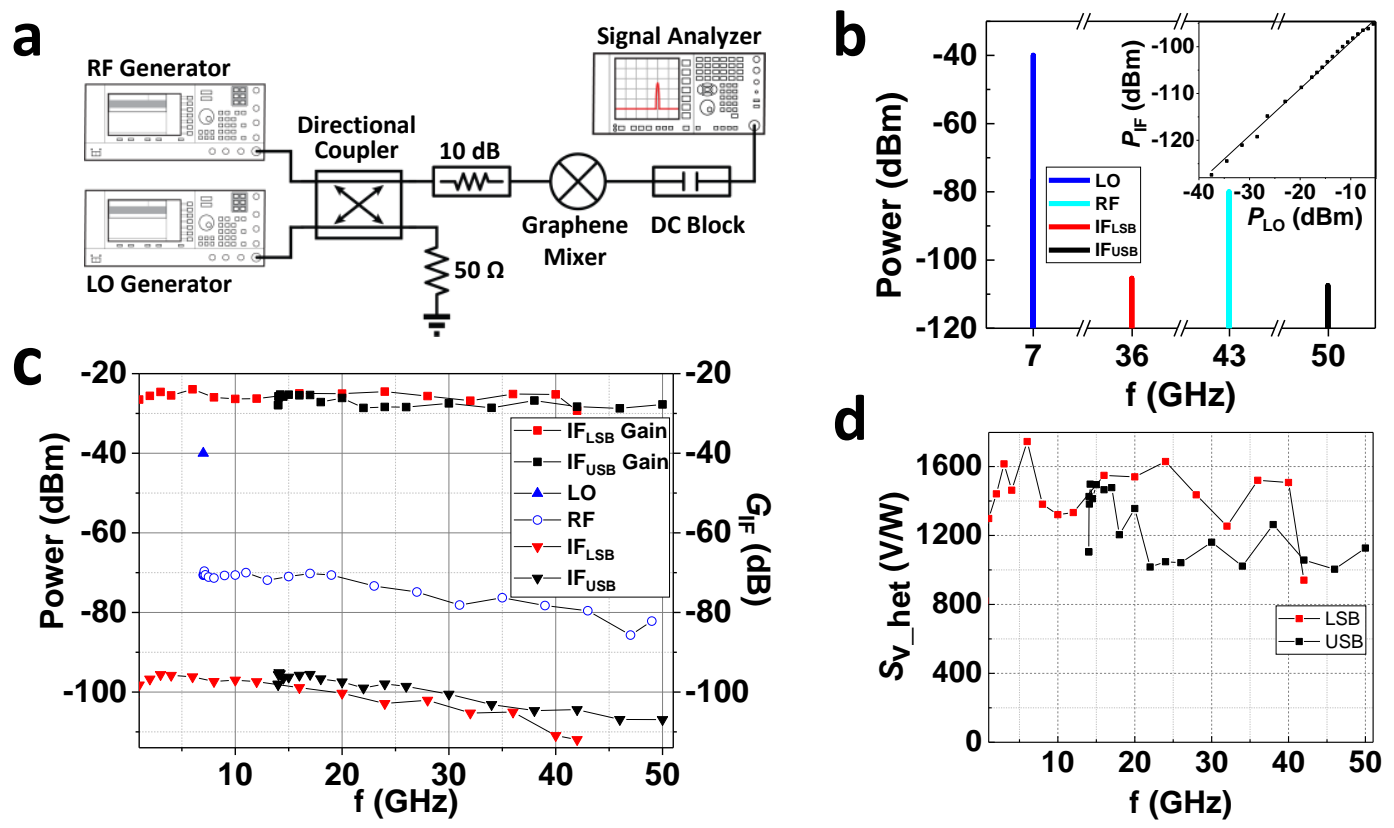

Figure 3. (a) RF heterodyne detection setup. (b) Graphene mixer spectrum with LO at $7 \mathrm{GHz}$ and RF at $43 \mathrm{GHz}$. Inset: IF power as a function of input LO power (the solid line is the linear fit with a fixed slope of 1). (c) Graphene RF mixer bandwidth/speed measurement. The red and black squares are for the conversion gains (right axis). The other symbols are for the transmitted LO, RF, IFLSB and IFUSB powers (left axis). (d) Device responsivity assessed from heterodyne mixing.

In Fig. $3 \mathrm{~b}$ we show a typical heterodyne mixing spectrum. In this measurement, the LO is set at $7 \mathrm{GHz}$ and the RF at $43 \mathrm{GHz}$; the LSB appears at $36 \mathrm{GHz}$ and the USB at 50 $\mathrm{GHz}$ as expected. It is interesting to note that the USB signal has a similar magnitude to the LSB signal, already suggesting that our mixer has very large bandwidth (the slightly smaller height is mostly due to system loss, not the frequency response of the graphene mixer; see below). 
Similar to the operation of a hot electron bolometric (HEB) mixer, ${ }^{30,31}$ the instantaneous power dissipated in graphene in the presence of LO and RF at different frequencies is given by:

$$
P=\frac{\left(V_{L O} \sin \left(2 \pi f_{L O} t\right)+V_{R F} \sin \left(2 \pi f_{R F} t\right)\right)^{2}}{R_{G}}
$$

Expansion of Eq. 2 results in terms oscillating at the IF frequencies that give rise to TE voltages $V_{I F}=2 \sqrt{P_{L O} P_{R F}} S_{V} \cos \left(2 \pi\left(f_{L O} \pm f_{R F}\right) t\right)$, where $P_{L O}=V_{L O}^{2} / 2 R_{G}$ is the input LO RMS power ( $P_{R F}$ is defined similarly). We then find the IF RMS power to be:

$$
P_{I F}=\left\langle\frac{V_{I F}^{2}}{R_{G}}\right\rangle=\frac{4 P_{L O} P_{R F} S_{V}^{2}\left\langle\cos ^{2}\left(2 \pi\left(f_{L O} \pm f_{R F}\right) t\right)\right\rangle}{R_{G}}=\frac{2 P_{L O} P_{R F} S_{V}^{2}}{R_{G}}
$$

or equivalently the conversion gain is:

$$
G_{I F}=\frac{P_{I F}}{P_{R F}}=\frac{2 S_{V}^{2} P_{L O}}{R_{G}}
$$

From Eq. 3, $P_{\mathrm{IF}}$ is expected to be proportional to $P_{\mathrm{LO}}$ at fixed RF power. We have tested this linearity and found that our device is linear over more than 3 decades of change in LO power; see Fig. 3b inset.

We then measured our graphene mixer over as wide a frequency range as allowed by our spectrum analyzer. Fixing the $\mathrm{LO}$ at $7 \mathrm{GHz}$, we tuned the RF from $8 \mathrm{GHz}$ to 50 GHz. Figure $3 \mathrm{c}$ shows the measured $\mathrm{IF}_{\mathrm{LSB}}$ and $\mathrm{IF}_{\mathrm{USB}}$ powers (down triangle, red and black) together with the LO (up blue triangle) and RF (open blue circle) power. We note that $\mathrm{IF}_{\mathrm{LSB}}$ and IFUSB powers are about $-95 \mathrm{dBm}$ at low frequencies and decrease to about $-110 \mathrm{dBm}$ at high frequencies; a similar $\sim 15 \mathrm{~dB}$ decrease of power is also observed for the RF. The similar IF and RF power decrease indicates that this roll-off is due to measurement system loss, not the frequency response of the graphene mixer. Indeed, using the definition in Eq. 
4 , we find the conversion gain of our graphene device to be $G_{\mathrm{IF}}=-27 \pm 3 \mathrm{~dB}$ over the whole $50 \mathrm{GHz}$ range (Fig. 3c, red and black squares).

This bandwidth is much larger than previous graphene RF mixers ${ }^{5,12,14,16}$ and is highly desirable for wideband applications. The wide bandwidth $(B)$ indicates that the thermal time constant of the heated electrons in graphene is shorter than $\tau=1 /(2 \pi B)=3$ ps. Similar high speed / wide bandwidths have been demonstrated in graphene mixer experiments with photons in the optical communication bands. ${ }^{19,20}$ We note that the wide IF bandwidth in all these cases is consistent with predictions that the intrinsic device speed for graphene detectors that rely on electron heating is at least $260 \mathrm{GHz}{ }^{32}$

We can also compare the bandwidth of our graphene mixer with that of HEB mixers. HEB mixers with $\mathrm{NbN}$ superconducting devices have been limited to about $4 \mathrm{GHz}$ bandwidth ${ }^{33}$ but recent results on $\mathrm{MgB}_{2}$ superconducting HEB mixers have increased this to about $10 \mathrm{GHz}{ }^{34}$ Two-dimensional electron gas (2DEG) HEB mixers, also operating at cryogenic temperatures, have demonstrated $40 \mathrm{GHz}$ bandwidth by exploiting ballistic transport. ${ }^{35}$

We can make use of the data in Fig. 3c and the mismatch loss measurement in Fig. $2 \mathrm{~b}$ to extract the voltage responsivity $S_{\mathrm{V}_{-} \text {het }}$ using Eq. 4 . As shown in Fig. 3d, we find $S_{\mathrm{V}_{-} \text {het }}$ $=1300 \pm 300 \mathrm{~V} / \mathrm{W}$ over $50 \mathrm{GHz}$ with experimental uncertainty less than $3 \mathrm{~dB}$, agreeing well with the DC measurements in Fig. 1d. This consistency validates our understanding of graphene heterodyne detection mechanism as described in Eqs. 2-4, and further confirms that the DC rectification provides a useful gauge to estimate device performance.

The fourth technique we use to measure the TE responsivity of our device is through $2^{\text {nd }}$ harmonic generation. Graphene is a material with an inversion center. From 
standard optical selection rules, $2^{\text {nd }}$ harmonic generation in graphene is forbidden while the $3^{\text {rd }}$ harmonic is allowed. ${ }^{22}$ Our device, however, displays the opposite behavior: in Fig. 4a, we excited our sample at $16 \mathrm{GHz}$, and observed that the $2^{\text {nd }}$ harmonic signal at $32 \mathrm{GHz}$ is more than two orders of magnitude stronger than the $3^{\text {rd }}$ harmonic signal at $48 \mathrm{GHz}$. We further performed power dependence in Fig. $4 \mathrm{~b}$ and found that the $2^{\text {nd }}$ harmonic signal indeed has quadratic input power dependence.
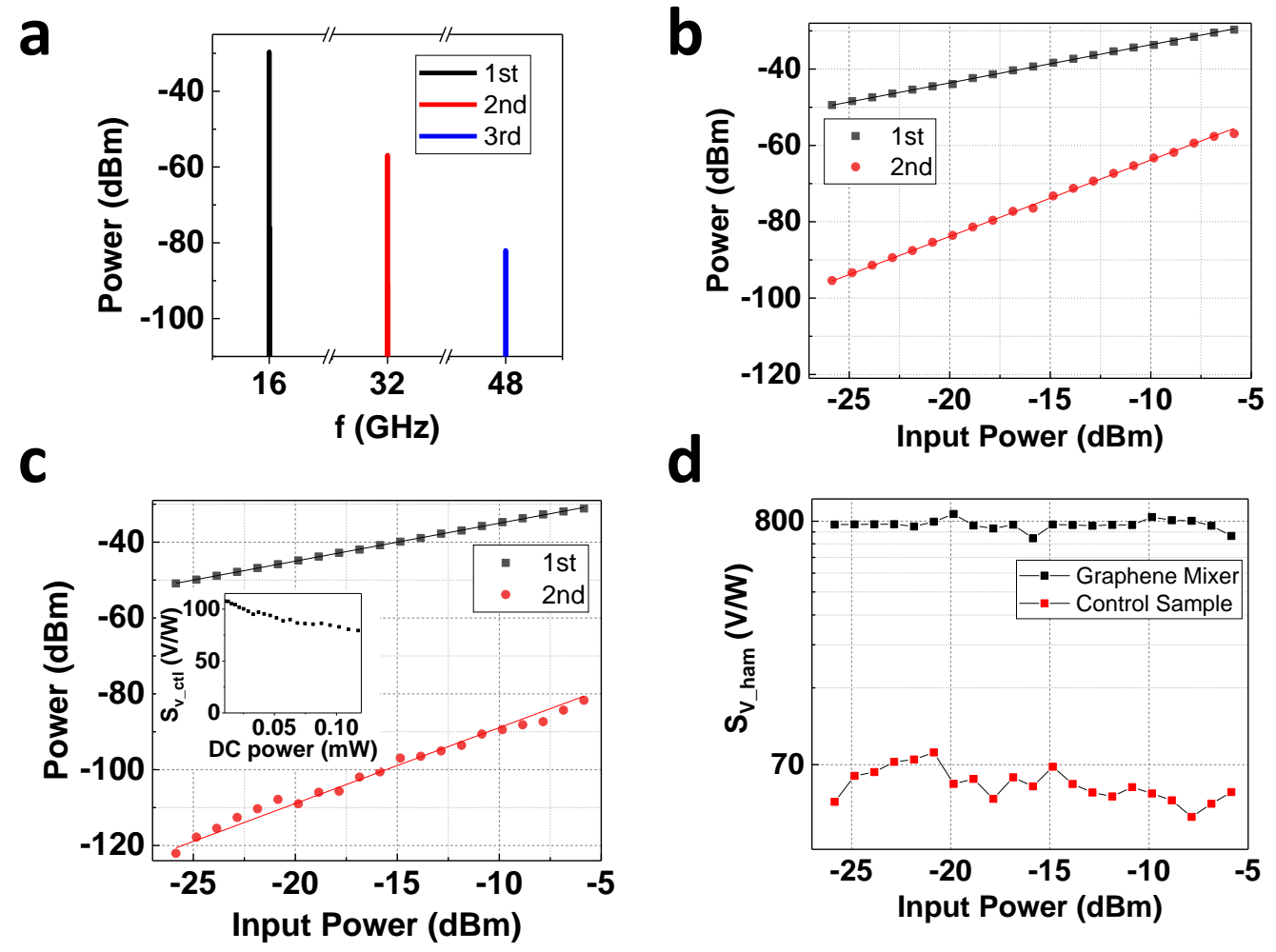

Figure 4. (a) The spectrum for harmonic signals generated in the graphene mixer device. The fundamental is set at $16 \mathrm{GHz}$. (b) Input power dependence of the 1st and 2nd harmonic signals; the solid lines are linear fits with fixed slopes of 1 and 2. (c) Same measurements as in (b) performed on the control sample. Inset: DC responsivity of the control sample. (d) Calculated responsivity from the secondharmonic conversion gain as a function of input power. 
While forbidden for standalone graphene, the appearance of a $2^{\text {nd }}$ harmonic signal is not so strange in light of the capability of our device to mix the RF and LO inputs. The $2^{\text {nd }}$ harmonic signal we observe can be viewed as the self-mixing of RF electric fields in the device due to the asymmetric contacts. To understand this phenomenon quantitatively, we revised the formulation in Eqs. 2-4. Here, the graphene is driven by MW at a single frequency and the instantaneous power is given by: $P=V^{2} \sin ^{2}(2 \pi f t) / R_{G}=V^{2}(1-$ $\cos (4 \pi f t)) / 2 R_{G}$. The second term is responsible for the $2^{\text {nd }}$ harmonic TE voltage: $V_{2 f}=$ $P_{f} S_{V} \cos (4 \pi f t)$. We find

$$
P_{2 f}=\frac{P_{f}^{2} S_{V}^{2}}{2 R_{G}}
$$

Similar to the application of Eq. 4 to find $S_{\mathrm{V}_{-} \text {het }}$, here we make use of Eq. 5 to extract $S_{\mathrm{V}_{-} \text {harm. }}$. This is shown in Fig. 4d (black squares). The extracted value of $S_{\mathrm{V}_{-} \text {harm }}=800 \pm 200 \mathrm{~V} / \mathrm{W}$ is in reasonable agreement with the values obtained from DC heating, RF heating, and RF mixing in Figures 1-3.

To further validate our understanding of this unusually strong $2^{\text {nd }}$ harmonic signal, we have fabricated a control device with similar DC resistance $(750 \Omega)$ but a smaller TE responsivity of about $90 \pm 20 \mathrm{~V} / \mathrm{W}$ (Fig. $4 \mathrm{c}$ inset). Interestingly its $2^{\text {nd }}$ harmonic signal is much smaller, about two orders of magnitude weaker. This can be understood from Eq. 5, where it is shown that the $2^{\text {nd }}$ harmonic intensity is expected to scale as $S_{V}{ }^{2}$. The two orders of magnitude weaker $2^{\text {nd }}$ harmonic generation is in excellent agreement with the control sample's $\sim 10$ times smaller $S_{\mathrm{V}}$. Lastly, we plotted the estimated $S_{\mathrm{V} \_ \text {harm }}$ for the control device in Fig. 4d (red squares), which gives $70 \pm 10 \mathrm{~V} / \mathrm{W}$, matching well with its DC responsivity in Fig. 4c inset.

The graphene RF device we developed here represents an important advance 
catching up with corresponding developments in the infrared optical frequencies. ${ }^{6,7,18-20}$ The device is operating efficiently as a direct detector, exhibiting an external responsivity as high as $130 \mathrm{~V} / \mathrm{W}(163 \mathrm{~mA} / \mathrm{W})$ over a large dynamic range, which is among the best for superfast graphene detectors. Heterodyne measurements demonstrated an intrinsic conversion loss of $27 \mathrm{~dB}$ and a bandwidth of more than $50 \mathrm{GHz}$, which are highly promising. It is of interest to compare our graphene mixer with existing technologies based on CMOS, SiGe and III-Vs (GaAs and InP). Currently CMOS technology is the most feasible for large scale systems and has the lowest cost. As was shown by Khamaisi et al., ${ }^{36}$ for $65 \mathrm{~nm}$ CMOS the conversion loss is in the range of 23-25.5 $\mathrm{dB}$ for LO frequencies from 220-300 GHz, comparable to our graphene mixer for intrinsic conversion loss. Bandwidths are 20-30 GHz, smaller than graphene. At shorter wavelengths beyond the transistor cutoff frequency $f_{\max }$, the conversion loss increases steeply. By incorporating LNAs (low-noise amplifiers) for RF and/or IF, more expensive integrated RF receivers have achieved better performances: SiGe, $15 \mathrm{~dB}$ conversion gain and $28 \mathrm{GHz}$ bandwidth (including a preamplifier) at $220 \mathrm{GHz} ;{ }^{37}$ GaAs metamorphic high electron-mobility transistors (mHEMTs), $3.5 \mathrm{~dB}$ gain and more than $10 \mathrm{GHz}$ bandwidth at $220 \mathrm{GHz} ;^{38} \mathrm{InP}$, the best performer, reaching $26 \mathrm{~dB}$ gain at $298 \mathrm{GHz}$ with a 3-dB bandwidth of $20 \mathrm{GHz} .{ }^{39}$ Our graphene RF mixer has larger bandwidth than the above technologies and has ample room for improvement in terms of operation frequency and conversion gain. The TE detection relies on absorption of RF power in a device with very low parasitic reactance. This lends its operation to translation to much higher frequencies, such as the sub-THz semiconductor devices as discussed above, ${ }^{36-39}$ as well as further up to several $\mathrm{THz}$, related to and improving upon the device we developed before. ${ }^{24}$ The conversion gain can be improved 
by applying a gate voltage,${ }^{24}$ lowering the device impedance and optimizing the device asymmetry. With its successful integration with $\mathrm{CMOS},{ }^{7}$ we envision that graphene RF mixers can be integrated with high performance RF and IF LNAs ${ }^{37-39}$ to further improve their performance, promising for emerging applications in high-speed communication systems at a few hundred $\mathrm{GHz},{ }^{40}$ as well as in thermal imaging systems in the $\mathrm{THz}$ range..$^{41,42}$

\section{Methods:}

\section{Sample fabrication.}

The detector devices were fabricated on CPW lines on a high resistivity silicon substrate with pads designed for microwave probing, see Fig.1 a-c. The CPW was fabricated following Ref. 43 in two steps: (1) pad frame and feed lines, (2) contact pads. The pad frame and coplanar feed-lines were defined by photolithography with $5 \mathrm{~nm}$ thick Ti and 80 $\mathrm{nm}$ thick $\mathrm{Au}$ deposited on the sample successively in an electron beam evaporator. The contact pads were then patterned in a similar way using photolithography with $1 \mu \mathrm{m}$ thick $\mathrm{Cu}$ and $500 \mathrm{~nm}$ thick $\mathrm{Al}$ deposited via magnetron sputtering. The fabricated structure consists of two GSG ports between which a $50 \Omega \mathrm{CPW}$ structure was formed. The graphene detector was placed in series with the Au center conductor of the CPW. The detector was fabricated with asymmetric contacts, similar (but not identical) to the device described in our work on a THz detector. ${ }^{24}$ The graphene and $\mathrm{hBN}$ samples we used in this study are all made by mechanical exfoliation. The graphene flake was confirmed to be monolayer by

Raman spectroscopy. ${ }^{44}$ We first made a BN/graphene/BN sandwich sample by the dry 
transfer method ${ }^{45}$ and then transferred the sandwich to the gap in between the central leads of the CPW, with graphene covering one side of the Au electrode. The other side of the sandwich is patterned with a two-layer PMMA mask, dry-etched using $\mathrm{CHF}_{3} / \mathrm{O}_{2}$ plasma and subsequently contacted to the left electrode with a graphene edge contact using palladium sputtering, similar to Ref. 23. This introduces asymmetry in the device that enables microwave detection and second-harmonic generation. The control sample is made by $\mathrm{hBN}$ covered graphene instead of a sandwich. To have similar resistance for easy comparison, the control sample's channel is about three times wider.

\section{RF direct detector measurements.}

We couple MW signals into the graphene device via bias-tees connected to GSG probes with $100 \mu \mathrm{m}$ pitch. The device is fed from a Keysight E8257D signal source, while the detected voltage is picked up on the center conductor and measured through two bias-tees (Anritsu K251) by a Keysight B2901A Precision Source/Measure Unit used as a voltmeter (see Fig.2a). The available MW power after the input bias-tee was measured with a Keysight N1913A power meter using a Keysight N8488A power sensor and was typically from $-28 \mathrm{dBm}$ to $-2 \mathrm{dBm}$. The signal generator output has an upper frequency of $50 \mathrm{GHz}$. By normalizing the thermoelectric voltage to the available input microwave power before the graphene device, we find the MW responsivity in Fig. 2a.

\section{RF heterodyne- and self-mixing measurements.}

For heterodyne measurements, the device is fed with LO and RF power at different $\mathrm{GHz}$ frequencies with two Keysight E8257D analog signal generators through a 3-dB RF 
directional coupler and a $10-\mathrm{dB}$ attenuator into the probe station. The $\mathrm{LO}$ power is set at $8 \mathrm{dBm}$ at the input of the device. The RF power is set $30 \mathrm{~dB}$ lower at $-38 \mathrm{dBm}$. The LO, RF and IF powers are measured from the output side of the device with a calibrated Keysight PXA spectrum analyzer. We verified that mixing of the LO and RF internal to the spectrum analyzer produces negligible IF power. For self-mixing measurements, we simply apply only one microwave signal to the detector through a $10-\mathrm{dB}$ attenuator and measure the transmitted power of the harmonic signal with the spectrum analyzer. The input power is swept from $-6 \mathrm{dBm}$ to $-26 \mathrm{dBm}$ at the device input at $16 \mathrm{GHz}$ for the two devices that were measured.

\section{Supporting Information}

$S$-parameter measurements of the device, the frequency dependence of the RF direct detector responsivity, and additional data on higher harmonic component measurements are found in the Supporting Information.

\section{Author information}

Corresponding Author:

†Jun Yan E-mail: yan@physics.umass.edu

ORCID

J. Yan: 0000-0003-3861-4633

\section{Notes}

The authors declare no competing financial interest.

\section{Acknowledgements}


This project was supported by the National Science Foundation under Grant Number ECCS-1509599. M. C. C. is supported by the Raytheon Integrated Defense Systems Engineering Advanced Studies Program Fellowship.

\section{References:}

(1) Bae, S.; Kim, H.; Lee, Y.; Xu, X.; Park, J.-S.; Zheng, Y.; Balakrishnan, J.; Lei, T.; Ri Kim, H.; Song, Y. Il; Kim, Y.-J.; Kim, K. S.; Özyilmaz, B.; Ahn, J.-H.; Hong, B. H.; Iijima, S. Roll-to-roll production of 30-inch graphene films for transparent electrodes. Nat. Nanotechnol. 2010, 5 (8), 574-578.

(2) Geim, A. K.; Novoselov, K. S. The rise of graphene. Nat. Mater. 2007, 6, 183191.

(3) Zhang, Z.; Du, J.; Zhang, D.; Sun, H.; Yin, L.; Ma, L.; Chen, J.; Ma, D.; Cheng, H.-M.; Ren, W. Rosin-enabled ultraclean and damage-free transfer of graphene for large-area flexible organic light-emitting diodes. Nat. Commun. 2017, 8, 14560.

(4) Liu, M.; Yin, X.; Ulin-Avila, E.; Geng, B.; Zentgraf, T.; Ju, L.; Wang, F.; Zhang, X. A graphene-based broadband optical modulator. Nature 2011, 474, 64-67.

(5) Lin, Y.-M.; Valdes-Garcia, A.; Han, S.-J.; Farmer, D. B.; Meric, I.; Sun, Y.; Wu, Y.; Dimitrakopoulos, C.; Grill, A.; Avouris, P.; Jenkins, K. A. Wafer-scale graphene integrated circuit. Wafer-scale graphene integrated circuit. Science 2011, 332 (6035), 1294-1297.

(6) Gan, X.; Shiue, R.-J.; Gao, Y.; Meric, I.; Heinz, T. F.; Shepard, K.; Hone, J.; Assefa, S.; Englund, D. Chip-integrated ultrafast graphene photodetector with high responsivity. Nat. Photonics 2013, 7, 883-887.

(7) Pospischil, A.; Humer, M.; Furchi, M. M.; Bachmann, D.; Guider, R.; Fromherz, 
T.; Mueller, T. CMOS-compatible graphene photodetector covering all optical communication bands. Nat. Photonics 2013, 7 (11), 892-896.

(8) Koswatta, S. O.; Valdes-Garcia, A.; Steiner, M. B.; Lin, Y.-M.; Avouris, P. Ultimate RF Performance Potential of Carbon Electronics. IEEE Trans. Microw. Theory Tech. 2011, 59 (10), 2739-2750.

(9) Schwierz, F. Graphene Transistors: Status, Prospects, and Problems. Proc. IEEE 2013, 101 (7), 1567-1584.

(10) Wang, H.; Hsu, A.; Wu, J.; Kong, J.; Palacios, T. Graphene-Based Ambipolar RF Mixers. IEEE Electron Device Lett. 2010, 31 (9), 906-908.

(11) Habibpour, O.; Vukusic, J.; Stake, J. A 30-GHz Integrated Subharmonic Mixer Based on a Multichannel Graphene FET. IEEE Trans. Microw. Theory Tech. 2013, $61(2), 841-847$.

(12) Han, S.-J.; Garcia, A. V.; Oida, S.; Jenkins, K. A.; Haensch, W. Graphene radio frequency receiver integrated circuit. Nat. Commun. 2014, 5, 3086.

(13) Habibpour, O.; He, Z. S.; Strupinski, W.; Rorsman, N.; Zirath, H. Wafer scale millimeter-wave integrated circuits based on epitaxial graphene in high data rate communication. Sci. Rep. 2017, 7, 41828.

(14) Andersson, M. A.; Zhang, Y.; Stake, J. A 185-215-GHz Subharmonic Resistive Graphene FET Integrated Mixer on Silicon. IEEE Trans. Microw. Theory Tech. 2017, 65 (1), 165-172.

(15) Generalov, A. A.; Andersson, M. A.; Yang, X.; Vorobiev, A.; Stake, J. A 400-GHz Graphene FET Detector. IEEE Trans. Terahertz Sci. Technol. 2017, 7 (5), 614 616. 
(16) Generalov, A. A.; Andersson, M. A.; Yang, X.; Vorobiev, A.; Stake, J. A heterodyne graphene FET detector at $400 \mathrm{GHz} .2017$ 42nd International Conference on Infrared, Millimeter, and Terahertz Waves (IRMMW-THz); IEEE, 2017; pp 1-2.

(17) Wells, J. Faster than fiber: The future of multi-Gb/s wireless. IEEE Microw. Mag. 2009, 10 (3), 104-112.

(18) Mueller, T.; Xia, F.; Avouris, P. Graphene photodetectors for high-speed optical communications. Nat. Photonics 2010, 4 (5), 297-301.

(19) Schall, D.; Neumaier, D.; Mohsin, M.; Chmielak, B.; Bolten, J.; Porschatis, C.; Prinzen, A.; Matheisen, C.; Kuebart, W.; Junginger, B.; Templ, W.; Giesecke, A. L.; Kurz, H. 50 GBit/s Photodetectors Based on Wafer-Scale Graphene for Integrated Silicon Photonic Communication Systems. ACS Photonics 2014, 1 (9), 781-784.

(20) Schuler, S.; Schall, D.; Neumaier, D.; Dobusch, L.; Bethge, O.; Schwarz, B.; Krall, M.; Mueller, T. Controlled Generation of a p-n Junction in a Waveguide Integrated Graphene Photodetector. Nano Lett. 2016, 16 (11), 7107-7112.

(21) Appleby, R.; Wallace, H. B. Standoff Detection of Weapons and Contraband in the $100 \mathrm{GHz}$ to 1 THz Region. IEEE Trans. Antennas Propag. 2007, 55 (11), 29442956.

(22) Kumar, N.; Kumar, J.; Gerstenkorn, C.; Wang, R.; Chiu, H.-Y.; Smirl, A. L.; Zhao, H. Third harmonic generation in graphene and few-layer graphite films. Phys. Rev. B 2013, 87 (12), 121406.

(23) Wang, L.; Meric, I.; Huang, P. Y.; Gao, Q.; Gao, Y.; Tran, H.; Taniguchi, T.; 
Watanabe, K.; Campos, L. M.; Muller, D. A.; Guo, J.; Kim, P.; Hone, J.; Shepard, K. L.; Dean, C. R. One-dimensional electrical contact to a two-dimensional material. Science 2013, 342 (6158), 614-617.

(24) Tong, J.; Muthee, M.; Chen, S.-Y.; Yngvesson, S. K.; Yan, J. Antenna Enhanced Graphene THz Emitter and Detector. Nano Lett. 2015, 15 (8), 5295-5301.

Cai, X.; Sushkov, A. B.; Suess, R. J.; Jadidi, M. M.; Jenkins, G. S.; Nyakiti, L. O.; Myers-Ward, R. L.; Li, S.; Yan, J.; Gaskill, D. K.; Murphy, T. E.; Drew, H. D.; Fuhrer, M. S. Sensitive room-temperature terahertz detection via the photothermoelectric effect in graphene. Nat. Nanotechnol. 2014, 9 (10), 814-819.

Zak, A.; Andersson, M. A.; Bauer, M.; Matukas, J.; Lisauskas, A.; Roskos, H. G.;

Stake, J. Antenna-integrated 0.6 THz FET direct detectors based on CVD graphene. Nano Lett. 2014, 14 (10), 5834-5838.

(27) Liu, L.; Hesler, J. L.; Xu, H.; Lichtenberger, A. W.; Weikle, R. M. A Broadband Quasi-Optical Terahertz Detector Utilizing a Zero Bias Schottky Diode. IEEE Microw. Wirel. Components Lett. 2010, 20 (9), 504-506.

(28) Yan, J.; Kim, M.-H.; Elle, J. A.; Sushkov, A. B.; Jenkins, G. S.; Milchberg, H. M.; Fuhrer, M. S.; Drew, H. D. Dual-gated bilayer graphene hot-electron bolometer. Nat. Nanotechnol. 2012, 7 (7), 472-478.

(29) Kim, M. H.; Yan, J.; Suess, R. J.; Murphy, T. E.; Fuhrer, M. S.; Drew, H. D. Photothermal response in dual-gated bilayer graphene. Phys. Rev. Lett. 2013, 110, 247402 .

(30) Yang, J.-X.; Agahi, F.; Dai, D.; Musante, C. F.; Grammer, W.; Lau, K. M.; Yngvesson, K. S. Wide-bandwidth electron bolometric mixers: a 2DEG prototype 
and potential for low-noise THz receivers. IEEE Trans. Microw. Theory Tech. 1993, $41(4), 581-589$.

(31) Karasik, B. S.; Elantev, A. I. Analysis of the Noise Performance of a HotElectron Superconducting Bolometer Mixer. Proc. 6th Intern. Symp. Sp. Terahertz Technol. 1995, 229-246.

(32) Urich, A.; Unterrainer, K.; Mueller, T. Intrinsic Response Time of Graphene Photodetectors. Nano Lett. 2011, 11 (7), 2804-2808.

(33) Cherednichenko, S.; Drakinskiy, V.; Berg, T.; Khosropanah, P.; Kollberg, E. Hotelectron bolometer terahertz mixers for the Herschel Space Observatory. Rev. Sci. Instrum. 2008, 79 (3), 34501.

(34) Novoselov, E.; Zhang, N. M.; Cherednichenko, S. MgB2 hot electron bolometer mixers for $\mathrm{THz}$ heterodyne instruments. International Society for Optics and Photonics, 2016, Vol. 9914, p 99141N.

(35) Lee, M.; Pfeiffer, L. N.; West, K. W. Ballistic cooling in a wideband twodimensional electron gas bolometric mixer. Appl. Phys. Lett. 2002, 81 (7), 12431245.

(36) Khamaisi, B.; Socher, E. Harmonic Down-Converters Around and Above the Cutoff Frequency. IEEE Trans. Microw. Theory Tech. 2015, 63 (7), 2275-2288.

(37) Ojefors, E.; Heinemann, B.; Pfeiffer, U. R. Subharmonic 220- and 320-GHz SiGe HBT Receiver Front-Ends. IEEE Trans. Microw. Theory Tech. 2012, 60 (5), $1397-1404$.

(38) Abbasi, M.; Gunnarsson, S. E.; Wadefalk, N.; Kozhuharov, R.; Svedin, J.; Cherednichenko, S.; Angelov, I.; Kallfass, I.; Leuther, A.; Zirath, H. Single-Chip 
220-GHz Active Heterodyne Receiver and Transmitter MMICs With On-Chip Integrated Antenna. IEEE Trans. Microw. Theory Tech. 2011, 59 (2), 466-478.

(39) Kim, S.; Yun, J.; Yoon, D.; Kim, M.; Rieh, J.-S.; Urteaga, M.; Jeon, S. 300 GHz Integrated Heterodyne Receiver and Transmitter With On-Chip Fundamental Local Oscillator and Mixers. IEEE Trans. Terahertz Sci. Technol. 2015, 5 (1), $92-$ 101.

(40) Abadal, S.; Alarcón, E.; Cabellos-Aparicio, A.; Lemme, M.; Nemirovsky, M. Graphene-enabled wireless communication for massive multicore architectures. IEEE Commun. Mag. 2013, 51 (11), 137-143.

(41) Chan, W. L.; Deibel, J.; Mittleman, D. M. Imaging with terahertz radiation. Reports Prog. Phys. 2007, 70, 1325-1379.

(42) Mittleman, D. Sensing with terahertz radiation; Springer, 2003.

(43) Pi, S.; Ghadiri-Sadrabadi, M.; Bardin, J. C.; Xia, Q. Nanoscale memristive radiofrequency switches. Nat. Commun. 2015, 6, 7519.

(44) Yan, J.; Zhang, Y.; Kim, P.; Pinczuk, A. Electric Field Effect Tuning of ElectronPhonon Coupling in Graphene. Phys. Rev. Lett. 2007, 98 (16), 166802.

(45) Chen, S.-Y.; Goldstein, T.; Tong, J.; Taniguchi, T.; Watanabe, K.; Yan, J. Superior Valley Polarization and Coherence of 2s Excitons in Monolayer WSe2. Phys. Rev. Lett. 2018, 120 (4), 46402. 
Table of Contents Graphic

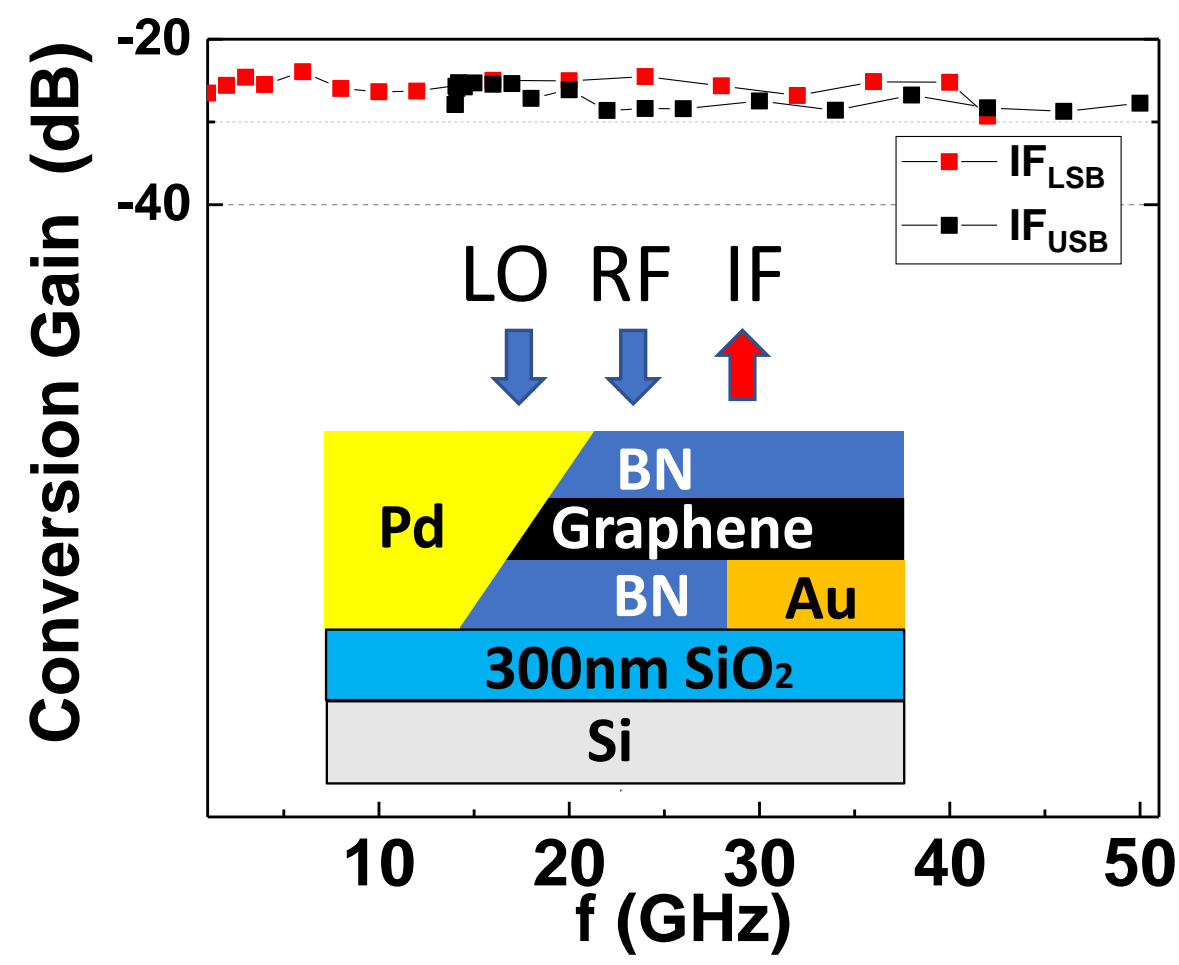

